\title{
Des vies sans fards : la représentation des femmes dans la littérature de jeunesse de Maryse Condé
}

https://doi.org/10.29173/af29432

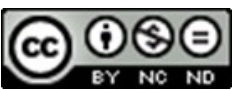

\section{Pauline Franchini}

pauline.franchini@u-bourgogne.fr

Université de Bourgogne, France

Résumé. Cet article propose une étude de la représentation des femmes dans trois romans de Maryse Condé pour la jeunesse: Rêves amers (réédition de Haïti chérie), Savannah Blues et La Belle et la Bête, une version guadeloupéenne. Dans ces fictions, Maryse Condé bouscule les stéréotypes de genre, en particulier autour de la maternité et de la figure de la potomitan ou la mère-courage antillaise. Elle interroge aussi les préjugés sexistes et racistes hérités du colonialisme et de l'esclavagisme, comme l'imagerie de la "mulâtresse », la belle métisse des îles. À travers l'analyse de personnages communs à ses romans pour la jeunesse et pour les adultes (Ségou et Desirada), l'étude montre que la littérature enfantine de Maryse Condé semble a priori chercher à édulcorer ou passer sous silence certaines violences sexuelles. Mais cette apparente autocensure ne doit pas masquer la critique et la remise en question audacieuse, dans ses romans pour jeune public, de l'intersectionnalité des dominations de race, de classe et de genre.

Mots clés : littérature de jeunesse; littérature antillaise; études postcoloniales; études de genre; double lectorat

Abstract. This article proposes a study of the representation of women in three of Maryse Condés novels for young people: Rêves amers (reprint of Haïti chérie), Savannah Blues and La Belle et la Bête, a Guadeloupean version. In these fictions, Maryse Condé challenges gender stereotypes, particularly around motherhood and the figure of the potomitan or West Indian mother-courage. She also questions sexist and racist prejudices inherited from colonialism and slavery, such as the imagery of the "mulatto", the beautiful mixed-race woman of the islands. Through the analysis of characters common to her novels for children and adults (Ségou and Desirada), the study shows that Maryse Condé's children's literature seems a priori to seek to water down or pass under silence certain sexual violence. But this apparent self- 
censorship should not obscure the bold critique and questioning of the intersectionality of race, class, and gender dominance in her novels for young readers.

Keywords: Children's literature; West Indian literature; postcolonial studies; gender studies; dual readership

$\mathrm{B}$ ien qu'elle se défende d'être féministe ${ }^{1}$, Maryse Condé place la voix des femmes ou leurs silences au cœur de ses préoccupations. Elle publie en 1979 La Parole des femmes : Essai sur les romancières des Antilles de langue française, dans lequel elle opère une typologie des

personnages féminins chez des autrices de la Caraïbe francophone et fournit une réflexion sur la condition féminine aux Antilles. Dans ses propres romans, Maryse Condé bouscule les stéréotypes de genre, en particulier autour de la maternité. Elle interroge aussi les préjugés sexistes et racistes hérités du colonialisme et de l'esclavagisme. À l'instar d'autres romancières guadeloupéennes telles que Simone Schwarz-Bart et plus récemment Gisèle Pineau, elle propose dans ses récits des portraits nuancés de femmes africaines et afro-caribéennes, qui dépeignent la « double colonisation » que subissent les femmes dans des sociétés patriarcales et postcoloniales (Petersen and Rutherford 3). Ses romans, qui mettent en scène le plus souvent le point de vue d'un personnage féminin à la première ou à la troisième personne, « cherchent à donner la parole à des voix qui n'ont pas toujours été entendues » (« seek to give expression to voices that have not always been heard ») comme l'écrit Simone A. James Alexander dans une étude consacrée à l'imaginaire maternel dans les romans des écrivaines afro-caribéennes (2). De fait, la critique postcoloniale et les études sur le genre ont fourni ces dernières années de très nombreux travaux sur les personnages féminins et la représentation des violences liées au genre dans l'œuvre de Maryse Condé, c'est-à-dire dans ses romans pour les adultes, puisque la recherche sur ses fictions pour la jeunesse est encore balbutiante.

Or, comme ce numéro spécial d'Alternative Francophone s'emploie fort justement à le souligner, Maryse Condé a aussi écrit des textes de fiction pour la jeunesse s'adressant pour la plupart à des élèves de collège, dont plusieurs collaborations avec le magazine « Je Bouquine » des éditions Bayard. Ces récits s'inscrivent le plus souvent dans le genre du roman réaliste - à l'exception de La Planète Orbis, un roman de science-fiction - et traitent de sujets sociaux contemporains touchant aux peuples de la Caraïbe et à leur diaspora, comme l'esclavage, le racisme ou l'exil. Dans un entretien datant de 2013, Maryse Condé confiait : «Un auteur écrit toujours le même livre, dit toujours les mêmes choses; on fait cela de façon différente, dans des cadres différents, mais, finalement, on parle un peu toujours du même sujet. » («Entretien avec Maryse Condé » 7). S'il n'est pas spécifiquement question de sa production pour la jeunesse dans cet extrait, on ne peut s'empêcher d'élargir l'idée d'une répétition « de façon différente, dans des cadres différents » à ses fictions pour les jeunes, publiées dans des contextes très différents des romans pour adultes. Tandis que les relations entre les sexes et les violences faites aux femmes, mais aussi aux très jeunes filles en contexte colonial et postcolonial sont des sujets récurrents dans les romans pour les adultes de Maryse Condé, font-ils l'objet de la même attention dans ses publications pour la

\footnotetext{
${ }^{1}$ Pour une analyse des entretiens dans lesquels Maryse Condé déclare sa méfiance à l'égard de l'étiquette de féministe, nous renvoyons à l'article de Julie Brugier « "Une féministe ! Qu'est-ce que c'est que cela ?" Penser le féminisme et l'écriture féminine dans Moi, Tituba sorcière... Noire de Salem (1986) et Célanire cou-coupé (2001) de Maryse Condé ».
} 
jeunesse? Peut-on observer des points de convergence ou au contraire une différence radicale dans les représentations des femmes et des mères?

Dans cette étude, nous aimerions montrer que, si Maryse Condé adapte la représentation de la violence à l'âge de son lectorat, il n'en demeure pas moins que ses récits pour jeune public proposent une lecture critique des hiérarchies de race, de classe et de genre et abordent la dimension intersectionnelle de l'oppression, pour reprendre le concept mis au jour par Kimberle Crenshaw (279). À travers l'analyse de personnages communs à ses romans pour la jeunesse et pour les adultes, nous montrerons dans un premier temps comment la littérature enfantine de Maryse Condé semble a priori chercher à édulcorer ou passer sous silence certaines violences sexuelles en opérant des modifications surprenantes. Mais cette apparente autocensure ne doit pas masquer la relecture et la remise en question audacieuse de quelques archétypes féminins dans ses romans pour jeune public. Savannah Blues montre une mère épuisée et défaillante; Madame Zéphyr dans Rêves amers et Mrs Jackson dans Savannah Blues représentent la caste mulâtre enrichie qui reproduit les oppressions de race et de classe sur ses domestiques à Haïti comme dans le Deep South; enfin, La Belle et la Bête, une version guadeloupéenne questionne entre les lignes l'érotisme et l'exotisme attachés à la figure de la femme des îles.

\section{PRATIQUES D’AUTO-RÉÉCRITURE ET EFFACEMENT DES VIOLENCES SEXUELLES}

Il est possible de constater un effacement des scènes ou allusions aux violences sexuelles en comparant des œuvres pour les adultes et pour les jeunes dans lesquelles on retrouve des personnages portant le même nom et ayant la même fonction. Ces migrations de personnages d'un roman à l'autre, mais dans une version nettement édulcorée pour la jeunesse soulèvent des interrogations quant à la réticence de Maryse Condé à aborder les violences faites aux femmes.

Ainsi, dans La Belle et la Bête, une version guadeloupéenne, de 2013, le père de Bella, une jeune guadeloupéenne métisse, est un bijoutier italien du nom de Gian Carlo Servandoni, gérant de la boutique Il Lago di Como. Ce détail, qui paraîtra insignifiant pour l'enfant, a de quoi troubler le lecteur ou la lectrice adulte du roman Desirada paru en 1997, dans lequel Marie-Noëlle pense être l'enfant illégitime que sa mère Reynalda aurait eue à l'âge de quatorze ans avec Gian Carlo Coppini, un Italien propriétaire de la bijouterie Il Lago di Como en Guadeloupe, au service duquel sa grand-mère Nina travaillait comme employée domestique. Marie-Noëlle, en quête de la vérité sur ses origines, se trouve confrontée à des versions contradictoires de la même histoire. Selon Reynalda, Nina, servante et maîtresse de Gian Carlo, était complice des viols commis par son patron et amant sur sa fille adolescente. Marie-Noëlle serait le fruit de ce viol. Dans cette version, Gian Carlo Coppini exerce sur sa victime une domination liée à la fois au sexe, à l'âge, à la position sociale et raciale : c'est un Européen blanc plutôt aisé, propriétaire d'un commerce, qui abuse de femmes noires qui lui sont hiérarchiquement inférieures et occupent des emplois subalternes à son service. Or, dans le roman pour adolescents, la mère de Bella s'appelle Madame Servandoni. Bella est donc l'enfant légitime de deux époux. Il n'est plus question de rapport de pouvoir au sein de ce couple, et encore moins de viol. De plus, dans la version « jeunesse », l'absence du père est justifiée par sa mort tragique, ce qui rapproche Bella de l'archétype de la pauvre orpheline de conte de fées, évacuant ainsi une thématique omniprésente dans la littérature caribéenne féminine, comme le note Kathleen Gyssels : «La maternité comme fardeau, les grossesses maudites, les enfants indésirés est certes

Alternative francophone

https://journals.library.ualberta.ca/af/index.php/af 
un des thèmes majeurs, on le sait, dans la littérature de la diaspora noire féminine. [...] Enfanter sans mariage, materner sans conjoint, sans contrat, prend pour nos auteures le même poids tragique. » (55).

Il serait alors possible de conclure dans un premier temps que, pour Maryse Condé, la représentation des violences sexuelles à l'intersection des dominations de genre, de race et de classe constitue la frontière à ne pas franchir entre littérature générale et littérature de jeunesse. Toutefois, dans Desirada, la version selon laquelle le bijoutier italien a abusé de la fille de sa servante provient avant tout d'un « souvenir imaginaire » (60), des rêves et de l'imagination de Marie-Noëlle, des termes répétés à de nombreuses reprises dans le roman : « Marie-Noëlle rêvait sa mère. Elle la rêvait avec tendresse et pitié. [...] Son imagination l'emportait entièrement. » (83). C'est finalement la version de Nina, la grand-mère, qui contrarie le scénario qui apparaissait jusque-là comme le plus plausible : "Tu as l'air déçue, toute chagrinée. Ce n'est pas cette histoire-là que tu avais envie d'entendre, pas vré? » (202) S'il est toujours possible que Nina mente pour protéger son ancien amant et pour se protéger elle-même de la justice, ses paroles suggèrent que le récit de l'adolescente violée et enceinte du patron blanc de sa mère domestique est une « histoire » qui plaît à l'imagination, car c'est l'histoire à laquelle on s'attend. Marie-Noëlle ellemême finit par douter en se rappelant que sa mère est écrivaine et que son métier consiste à inventer des histoires : «Car c'est une de ces histoires, trop belles pour être vraies avec lesquelles on écrit des romans. Dans le fond, elle est un écrivain, ma mère, et elle a bâti sa fiction. » (252). De fait, la littérature des autrices caribéennes et afro-américaines accorde une large place aux personnages de femmes subalternes, opprimées et abusées par le maître ou le patron blanc, qu'il s'agisse des slaves narratives ou des récits dont l'intrigue se situe après l'abolition de l'esclavage ${ }^{2}$. En refusant de confirmer une version semblable aux histoires « avec lesquelles on écrit des romans », Maryse Condé esquive le scénario attendu et refuse une fois de plus de se laisser enfermer dans une catégorie et une identité qu'elle récuse, telle que « féministe » ou « écrivaine antillaise ». Dans ces conditions, le fait que le roman La Belle et la Bête refuse la trame la plus stéréotypée, à savoir la prise de pouvoir de Gian Carlo sur la mère Bella, ne relève pas uniquement de l'autocensure ou de l'édulcoration liée à l'édition pour la jeunesse. On peut aussi y voir un énième pied de nez à la critique postcoloniale et féministe, typique de la posture d'écrivaine singulière et inclassable que Maryse Condé cultive à longueur d'entretiens. Qu'elle s'adresse aux adultes ou aux plus jeunes, l'œuvre de Maryse Condé, comme l'écrit Yolaine Parisot, « s'appliqu[e] tout particulièrement à déjouer les horizons d'attente $»^{3}(132)$. La disparition du thème de la violence sexuelle dans le roman pour la jeunesse serait alors non pas la borne-frontière entre les deux lectorats de Maryse Condé, mais au contraire un signe de la cohérence de l'ensemble de son œuvre.

En revanche, le roman historique sur l'esclavage Chiens fous dans la brousse permet de mesurer de façon plus évidente encore l'effacement de la représentation des violences liées au genre dans le processus de réécriture d'une intrigue antérieure pour un jeune public. Les lieux et la trame de Chiens fous dans la brousse de 2008 (d'abord paru chez « Je Bouquine » en 2006) ne sont pas inédits : les lecteurs et lectrices adultes auront pu les rencontrer auparavant dans le roman Ségou: Les Murailles de terre, premier tome de la grande fresque historique africaine de Maryse Condé. Dans sa bibliographie, Chiens fous dans la brousse est un cas unique d'auto-réécriture en version enfantine. En effet, la quasi-intégralité des actions relatées dans le roman pour la jeunesse correspondent à des scènes déjà écrites dans le roman antérieur pour les adultes. L'autrice a isolé et adapté le destin de trois personnages de Ségou - Naba, Malobali et

\footnotetext{
${ }^{2}$ Dans Pluie et vent sur Télumée Miracle de Simone Schwarz-Bart, le béké M. Desaragne s'introduit dans la petite chambre de bonne et s'arroge sur sa jeune domestique noire un droit de cuissage qui lui semble tout naturel, hérité des pratiques de ses ancêtres esclavagistes.

${ }^{3}$ Dans Pluie et vent sur Télumée Miracle de Simone Schwarz-Bart, le béké M. Desaragne s'introduit dans la petite chambre de bonne et s'arroge sur sa jeune domestique noire un droit de cuissage qui lui semble tout naturel, hérité des pratiques de ses ancêtres esclavagistes.
} 
Ayodélé - pour en faire un roman pour les dix-quatorze ans sur les rapts d'enfants au Mali par des trafiquants d'esclaves au dix-huitième siècle ${ }^{4}$.

Dans Chiens fous dans la brousse, les protagonistes sont plus jeunes que dans Ségou, et la relation amoureuse entre Naba et Ayodélé est gommée au profit d'un sentiment fraternel. Le capitaine du bateau négrier qui les amène au Brésil est aussi un personnage commun au roman pour les adultes et au roman pour la jeunesse. Dans Ségou, cet homme cruel et cynique ne fait pas mystère du sort d'esclave sexuelle qui attend Ayodélé à la plantation, ce que confirmeront les événements de la suite du roman : "Quant à la fille, étant donné son joli minois et son jeune âge, elle ne tarderait pas à devenir "maîtresse de maison" et à mettre au monde des bâtards métis. » (124). Or, il est intéressant de relever que dans la scène correspondante dans Chiens fous dans la brousse, le capitaine du bateau prédit qu'Ayodélé et les autres enfants seront affectés à la cueillette du café en raison de la petite taille de leurs mains. Dans sa description physique comme dans son rôle dans la diégèse, le personnage d'Ayodélé est totalement désexualisé dans le roman pour la jeunesse. L'intrigue se concentre sur la thématique de l'esclavage sans la complexifier davantage en ajoutant à la présentation de la domination coloniale et raciale celle de la domination sexuelle.

Au-delà des pratiques d'auto-réécriture et des doublons de personnages, on observe d'une façon générale chez Maryse Condé une réticence à aborder la question des violences sexuelles en littérature de jeunesse. Dans Rêves amers ${ }^{5}$, Rose-Aimée, une jeune Haïtienne de treize ans durant la dictature de Jean-Claude Duvalier, est envoyée à Port-au-Prince pour travailler comme domestique, forme d'esclavage moderne, avant d'embarquer clandestinement pour la Floride avec son amie Lisa et d'autres boat-people. Alors que les deux adolescentes sont contraintes de passer plusieurs nuits dans la rue et doivent réunir de l'argent et négocier le prix de la traversée avec les passeurs, elles ne sont impliquées dans aucune situation d'agression ou de chantage sexuel. La question transhistorique et transnationale du viol n'étant pas spécifique à la période et au lieu décrits, tout se passe comme si l'autrice refusait d'alourdir un récit abordant des problématiques culturelles, historiques et sociales aussi complexes - dictature, misère et exil à Haïti sur fond d'histoire coloniale et esclavagiste. Ici, le fait de mettre en scène de jeunes héroïnes n'apporte pas à l'action un enjeu supplémentaire qui pourrait faire l'objet d'une analyse par le prisme du genre. Les protagonistes auraient pu tout aussi bien être deux garçons sans qu'aucune scène ne soit modifiée.

Ce que l'on peut qualifier d'autocensure ou plus justement de choix didactique stratégique, dans la mesure où il s'agit de focaliser l'attention des jeunes lecteurs et lectrices sur une thématique, par exemple l'esclavage, conduit donc Maryse Condé à atténuer ou supprimer la dimension intersectionnelle et genrée des violences et à passer sous silence la question spécifique de la condition féminine dans le cadre d'une oppression plus large dans ses récits pour jeune public. Pour autant, il serait faux de penser que Maryse Condé n'a pas à cœur de déconstruire certains stéréotypes de genre dans ses romans pour la jeunesse, à commencer par le mythe puissant de la mère aimante et protectrice.

\footnotetext{
${ }^{4}$ Pour une analyse comparée détaillée, voir l'article «De Ségou à Chiens fous dans la brousse : un exemple d'autoréécriture en version enfantine chez Maryse Condé » (Franchini 2020).

${ }^{5}$ Rêves amers est la réédition de Haïti chérie, d'abord paru dans « Je Bouquine » en 1987 puis en poche en 1991 chez Bayard Jeunesse.
}

Alternative francophone

https://journals.library.ualberta.ca/af/index.php/af 


\section{SAVANNAH BLUES OU L'EFFONDREMENT DU «POTOMITAN »}

Des siècles d'esclavage et de colonisation ont laissé des cicatrices profondes dans la société caribéenne et en particulier dans les liens familiaux et dans les relations entre les hommes et les femmes. Selon Bonnie Thomas, l'un des effets de l'histoire coloniale et esclavagiste des Amériques a été l'émergence d'une société majoritairement matrifocale dans laquelle le rôle de la mère élevant seule ses enfants devient central (16). Certes, les familles matrifocales et la violence domestique et sexuelle étaient déjà des thèmes récurrents dans la littérature antillaise produite par des hommes avant l'émergence des femmes écrivaines dans les années 1970. Le grand nombre de personnages féminins victimes de viol qui font figure de «mères courage » fortes et résilientes s'explique par la volonté des écrivains nationalistes de dénoncer l'oppression coloniale à travers les souffrances du corps féminin et maternel profané qui devient l'allégorie de l'île natale conquise et colonisée (Rody 113). C'est une façon pour les auteurs guadeloupéens et martiniquais de contrer le discours colonial qui érige la France en mère patrie, en y opposant l'image d'une île-mère longtemps violentée, mais toujours debout. Pour Caroline Rody, ce « récit phallocentrique du nationalisme » (« phallocentric narrative of nationalism ») qui a dominé le canon littéraire antillais jusqu'aux années 1970 « avait tendance à réifier les femmes et circonscrire les possibles de leurs représentations » (« tended to objectify women and delimit their figural possibilities », 112). On retrouve par exemple ce stéréotype de la grand-mère sacrificielle, aimante et dévouée, dans $L a$ Rue Cases-Nègres de Joseph Zobel en 1950, avec le personnage de M'man Tine.

Par la suite, les autrices caribéennes inventent quant à elles des « définitions non-traditionnelles de la maternité » («non-traditional definitions of motherhood») (Thomas 15) qui contrebalancent cette figure maternelle idéalisée issue à la fois de la culture chrétienne occidentale et de la tradition littéraire nationaliste et masculine de la Caraïbe qui valorise l'image de la mère " potomitan » (littéralement le « poteau du milieu » en créole, qui permet à la case de ne pas s'effondrer), infatigable cheffe de famille et solide pilier du foyer. Kathleen Gyssels identifie même un « archétype de la mauvaise mère » chez Maryse Condé comme chez Toni Morrison (35). Pour Caroline Rody, la littérature caribéenne féminine s'empare de l'image de l'île-mère et du thème de la maternité d'une façon différente, dans la mesure où elle dépeint plus volontiers des relations conflictuelles entre mères et filles, dans des intrigues qui s'attachent moins à la représentation du corps maternel violenté qu'aux rapports douloureux entre femmes de la même lignée (124). En effet, les romans d'autrices comme Maryse Condé, Gisèle Pineau ou Edwidge Danticat (cette dernière pour la littérature haïtienne-américaine anglophone), abondent en personnages de femmes épuisées, fragilisées, qui baissent les bras, abandonnent leur devoir parental et perçoivent la maternité comme une malédiction, très loin du stéréotype de la manman créole, la mèrecourage considérée comme une femme forte et une héroïne (Roch 108).

On pourrait penser que la littérature adressée à l'enfance et à la jeunesse privilégie par défaut des intrigues avec des structures familiales aimantes et protectrices et surtout des figures de mères, sinon parfaites, du moins qui assurent de leur mieux leur « travail» de maman ${ }^{6}$. Lorsque ce n' est pas le cas, la défaillance parentale ou maternelle est une anomalie qui constitue la thématique centrale du roman, que l'on songe par exemple aux fictions pour la jeunesse consacrées à des sujets sociétaux très ciblés comme la maladie, la dépression ou les addictions d'un parent, ou encore la maltraitance intrafamiliale ${ }^{7}$. Or, le roman pour la jeunesse Savannah Blues, d'abord publié dans « Je Bouquine » en 2004, puis aux éditions

\footnotetext{
${ }^{6}$ On peut songer à la mère maladroite, tête en l'air, éternelle adolescente mais attachante et complice dans la série de bandes-dessinées pour la jeunesse Lou! de Julien Neel.

${ }^{7}$ Voir par exemple le roman Solaire de Fanny Chartres (L'École des Loisirs, 2018) qui aborde le thème de la dépression d'une mère après son divorce et ses conséquences sur les enfants livrés à eux-mêmes.
} 
Sépia en 2009, présente un personnage de mère faillible, sans que la narration ne porte de jugement de valeur et sans que cet aspect de l'intrigue en soit la problématique centrale.

Savannah Blues se déroule dans les années 2000 en Géorgie, aux États-Unis, dans un quartier pauvre fortement touché par le chômage qui affecte principalement la population afro-descendante. Zora, treize ans, s'occupe seule de ses frères et de sa sœur. Son quotidien consiste à trouver de quoi les nourrir, les habiller et les empêcher de tomber dans la délinquance. La fratrie est livrée à elle-même après le départ de leur père au Canada pour trouver du travail et la dépression de leur mère Louise, qui ne quitte plus son lit, ne s'alimente plus et se laisse dépérir. Louise a perdu son emploi de femme de ménage dans un hôtel et s'est persuadée qu'elle ne reverra pas son mari vivant. Les rôles mère-fille s'inversent et l'adolescente prend soin de sa mère comme s'il s'agissait d'un petit enfant. Elle endosse le rôle d'adulte et cherche du travail dans un fast-food pour subvenir aux besoins de la famille. Personne dans le voisinage ne s'inquiète de leur sort jusqu'à l'arrivée des services de la protection de l'enfance.

Bien que cette mère laisse ses enfants déscolarisés, affamés et sans ressources, la narration s'abstient de tout commentaire négatif sur la négligence parentale et suggère plutôt avec compassion que c'est une femme vidée, à bout de forces, épuisée d'être forte : «Un ressort s'était rompu en elle » (93). Sans rentrer dans le détail des causes de sa dépression, le texte exprime sobrement : « Louise était trop malheureuse pour porter attention à d'autres souffrances que les siennes. » (24). L'image du ressort brisé figure en quelque sorte l'envers réaliste de l'idéalisation de la mère potomitan, ce solide pilier qui porte sa famille à bout de bras et qui n'a pas le droit de lâcher. Ici, le poteau central s'est écroulé sous le poids des soucis et des responsabilités. Maryse Condé semble avoir toute confiance en la capacité de son jeune lectorat à comprendre, sans en passer par de longues explications psychologiques, qu'il existe des circonstances dans lesquelles les femmes et les mères peuvent perdre pied et s'effondrer.

Or, il est intéressant de noter que cette structure familiale atypique impliquant la parentalisation précoce de la jeune héroïne et les difficultés économiques et sociales de son foyer ne constituent pas la problématique centrale du roman, ou du moins sont insérées dans une problématique plus vaste qui ne se situe pas à l'échelle du drame individuel. Ce n'est pas un hasard si le titre du roman fait référence à une pratique culturelle afro-américaine ancrée dans le Sud des États-Unis, le blues, et au nom de la ville de Savannah plutôt qu'au nom de l'héroïne Zora ${ }^{8}$. Savannah Blues traite avant tout du racisme et des inégalités persistantes qui gangrènent une région hantée par son histoire esclavagiste : il est question du blues d'une ville entière, et au-delà, de tout un pays, davantage que des émois personnels de l'adolescente. En effet, le roman insiste à de nombreuses reprises sur le passé esclavagiste de sa ville, appelée Middleton dans le roman, malgré un titre qui renvoie à la ville de Savannah en Géorgie. Zora connaît parfaitement l'histoire des planteurs et des esclaves de la ville de Middleton. Elle est fière de porter le prénom de Zora Neale Hurston, écrivaine et anthropologue spécialiste du folklore afro-américain et du vaudou haïtien, tandis que les prénoms de ses petits frères sont un hommage à Richard Wright et Langston Hughes. Son père est versé dans la culture rasta jamaïcaine, porte « des boubous brodés à

\footnotetext{
${ }^{8}$ Ce n'est pas la première fois qu'un titre de roman pour la jeunesse de Maryse Condé peut prêter à confusion, d'autant que la couverture des éditions Sépia présente une photographie d'enfant. Il faut lire Savannah Blues pour comprendre que Savannah n'est pas le prénom de la petite fille représentée en couverture. De la même façon, le héros du roman Hugo le terrible se nomme Michel, tandis que le titre fait référence au cyclone Hugo qui a touché les Antilles en 1989. Maryse Condé joue avec les codes de la littérature d'enfance et de jeunesse où les titres contiennent souvent le nom du héros éponyme représenté en couverture.
}

Alternative francophone

https://journals.library.ualberta.ca/af/index.php/af 
l'africaine » (21) et revendique fièrement une forme de négritude, tandis que le grand-père paternel de Zora, qui a connu la ségrégation et ses humiliations, conseille à son fils de « rester à sa place». Cet enracinement et cette intériorisation de la discrimination raciale troublent profondément la jeune fille :

Tu ne sais pas rester à ta place. Ce reproche tourmentait Zora qui ne cessait de se demander ce qu'il signifiait. Est-ce que chacun de nous reçoit au moment de sa naissance, une place immuable qu'il ne doit pas essayer de quitter? Les uns en haut de l'échelle sociale, les autres en bas? Les uns comblés de richesses matérielles, les autres les mains vides? (21)

De fait, malgré la fin de la ségrégation, les communautés blanche et noire de Middleton ne se mélangent pas. Une troisième classe intermédiaire, celle des « mulâtres », des Noirs à la peau claire qui jouissent de plus de privilèges matériels et symboliques que les autres en raison de la part blanche de leur ascendance, s'incarne dans le personnage de la voisine Agnès Jackson. Au travers de ce personnage, le roman aborde frontalement la complexité des hiérarchies sociales et raciales au sein même de la communauté afroaméricaine, des rapports hérités d'un passé esclavagiste récent, toujours douloureux, et aux conséquences bien palpables :

Agnès Jackson [...] appartenait à la bourgeoisie mulâtre constituée dans le Sud par les descendants des nombreux enfants que les planteurs avaient eus avec leurs esclaves noires. Légitimes ou pas, ces planteurs avaient doté leurs rejetons couleur café au lait de terres et de comptes en banque. [...] Louise soutenait que les bourgeois à peau claire du Sud des États-Unis méprisent tous ceux qui sont plus foncés qu'eux. (42-43)

Agnès Jackson avait commodément oublié que sa famille sortait du ventre d'une pauvre esclave noire, peut-être violentée par son planteur de maître. Elle choisissait de se souvenir seulement des politiciens, des avocats, des gouverneurs qui composaient sa descendance. Son cœur était complètement desséché par l'orgueil (59).

Agnès Jackson, femme métisse qui aime que sa cuisinière noire lui parle en baissant les yeux, rappelle Madame Zéphyr dans Rêves amers, riche « mulâtresse à la peau couleur de miel » (26), épouse d'un diplomate haïtien, qui brutalise ses domestiques et leur parle en utilisant le langage du colon : « Regardezmoi cette petite négresse, cette petite paresseuse qui dort! » (25). Maryse Condé semble ici donner une illustration des théories de Peau noire, masques blancs de Frantz Fanon (1952) à l'échelle des jeunes lecteurs et lectrices. Notons que c'est finalement auprès de son amie indienne Gayatri que Zora découvre qu'il est possible de se trouver des points communs avec celles et ceux qui ne partagent ni la même couleur de peau ni la même religion : « Mais de ces différences, il ne fallait pas avoir honte. Ne donnaient-elles pas la preuve de cette fascinante diversité du monde? » (118). La réaction de la jeune fille esquisse à hauteur d'enfant une poétique de l'ouverture à l'autre et de l'identité plurielle, composite et fluctuante, qui tire sa richesse des différences et de la créolisation des cultures; en somme, une poétique de la relation et du « Tout-Monde » qui rejoint les concepts forgés par Édouard Glissant.

On le voit, la question de l'identité et celle des inégalités raciales et sociales qui se perpétuent de génération en génération depuis l'esclavage sont au premier plan de l'intrigue de Savannah Blues. Louise, la mère, n'est pas tenue pour responsable à un niveau individuel de la situation dramatique dans laquelle se trouvent Zora, ses frères et sa sœur : c'est bien la société postcoloniale qui est mise en cause. Outre cette figure maternelle atypique, le personnage d'Agnès Jackson, sorte de double américain de Madame Zéphyr à Haïti, montre que Maryse Condé, fuyant le manichéisme et les oppositions binaires, n'hésite pas à présenter à son jeune lectorat les multiples facettes de la domination de genre, de race et de classe, en particulier dans le domaine du travail domestique, y compris au sein de la communauté noire : «Zora découvrait que servir les autres n'est pas agréable. Penser que Ma Dana s'était pendant toute sa vie louée comme cuisinière pour des mulâtres de Middleton. » (59). Mais c'est surtout dans La Belle et la Bête, une 
version guadeloupéenne que la question des hiérarchies raciales, sociales et sexuelles dans la société caribéenne postcoloniale est mise en exergue à travers le personnage de Bella, une jeune fille à la croisée des stéréotypes sexistes des hommes de sa communauté et de ceux de l'imaginaire colonial et raciste.

\section{«VOILÀ TON CAFÉ, MAÎTRE »: QUESTIONNER LE STÉRÉOTYPE DE «LA BELLE MULÂTRESSE » EN LITTÉRATURE DE JEUNESSE}

Recommandé pour les 13-14 ans, La Belle et la Bête, une version guadeloupéenne est d'une lecture difficile et exigeante qui s'accompagne de notes de bas de page et d'un dossier pédagogique. L'histoire se passe en 2005 avant le passage imminent de l'ouragan Katrina à Sangre Grande, un village de pêcheurs fictif de Guadeloupe touché par la pauvreté, le chômage et l'exil de masse de ses habitants. Le roman fait référence à des aspects historiques et culturels de la Caraïbe comme l'esclavage, l'art culinaire, la tradition du carnaval, mais traite aussi de sujets controversés tels que l'attitude néocoloniale de la France à l'égard de ses anciennes colonies du point de vue de certains personnages, les ravages du tourisme de masse, l'aliénation culturelle, l'alcoolisme. En plus de ce portrait complexe d'une situation de postcolonialité, le thème du sexisme et de l'hétéronormativité des relations entre les sexes à la Guadeloupe parcourt le roman de façon sous-jacente. Le titre renvoie ironiquement au conte de fées européen, dans la mesure où il s'agit d'un récit réaliste qui se termine par un mariage de convenance conclu dans un intérêt financier et par une vision désenchantée des relations hommes-femmes. Bella incarne la « double colonisation » de la femme afro-caribéenne à l'intersection des oppressions de race et de genre. En effet, en tant que jolie jeune femme métisse, elle est décrite uniquement comme l'objet du désir masculin dans une société patriarcale. De plus, en tant que fille d'un Italien blanc et d'une Guadeloupéenne noire, elle est rejetée par la famille raciste de son père.

Au début du roman, Carmélien est amoureux de la belle et arrogante Bella, bien qu'il sache qu'il n'a aucune chance de la séduire à cause de son apparence physique disgracieuse. Pensant que son fils ne trouvera jamais de femme, la mère du jeune homme lui apprend à cuisiner et entretenir une maison, souscrivant à la répartition traditionnelle des rôles genrés selon laquelle les tâches ménagères incombent normalement aux femmes : " Il savait tout faire par lui-même, laver son linge, empeser ses chemises, repasser ses pantalons, car Victorine, sa mère, avait veillé au grain. » (14). En tant que garçon né et élevé dans une société patriarcale, Carmélien envisage la possibilité d'avoir recours à la magie du quimbois ${ }^{9}$ antillais pour ensorceler Bella afin de la rendre amoureuse de lui et faire d'elle son esclave, plutôt que de se résoudre à ne pas pouvoir obtenir son consentement : " il aurait pu charmer Bella, faire d'elle un zombie qui obéirait à toutes ses volontés et n'aurait qu'un désir, celui de lui plaire. » (18). Cela fait écho au prénom même de Carmélien qui évoque le sort, l'enchantement (du latin carmen, carminis) : à défaut d'être un prince charmant de contes de fées, celui qu' on surnomme La Bête songe à user d'un charme magique. Il considère tout naturellement que la satisfaction de ses propres désirs l'emporte sur le consentement de la jeune femme.

Cette dialectique maître-esclave se poursuit lorsque Carmélien rêve à sa vie idéale aux côtés de Bella : « l'air doux et soumis, Bella allait et venait, vêtue d'un tablier blanc. Elle tenait entre ses mains une tasse

${ }^{9}$ Aux Antilles, selon les croyances traditionnelles, le quimboiseur ou la quimboiseuse maîtrise la médecine par les
plantes, communique avec les esprits, pratique des envoûtements ou peut vous délivrer d'un sort qui vous a été jeté.

Alternative francophone

https://journals.library.ualberta.ca/af/index.php/af 
de porcelaine posée sur une soucoupe. - Voilà ton café, maître, est-il assez sang de taureau comme tu l'aimes? » (30) Cette vision de la femme comme esclave domestique de son mari correspond effectivement aux résultats de la vaste enquête sociologique de France Alibar et Pierrette Lembeye-Boy sur la condition féminine aux Antilles en 1990. Dans la littérature caribéenne féminine pour les adultes, on trouve des personnages d'épouses en situation de servitude domestique et sexuelle, comme Ninette dans La grande drive des esprits de Gisèle Pineau, archétype de la femme au foyer " douce et soumise » dont rêve Carmélien. Toutefois, la suite du roman offre un retournement de situation : c'est Carmélien qui se met au service de Bella. Ironiquement, il fait le ménage et la cuisine et lui apporte son café dans une inversion parfaite des rôles par rapport à son rêve de vie maritale. Cette attitude " féminine » selon les codes d'une société sexiste soulève d'ailleurs l'indignation de son ami Le Kid : "Te voilà l'esclave d'une femme! » (75). Plus tard, Le Kid désapprouve également le comportement de l'épouse d'un autre personnage : « Depuis quand est-ce que la femme commande à l'homme? » (90).

De telles remarques ne sont pas destinées à être prises au premier degré par les jeunes lecteurs et lectrices dans la mesure où l'attitude machiste des hommes est tournée en dérision tout au long du roman. Par exemple, les hommes du village se rassemblent au bar pour boire et écouter le discours d'Agénor, un politicien local. Agénor ouvre une école privée pour enseigner aux jeunes garçons l'art ancestral de la sorcellerie guadeloupéenne, le quimbois. Son discours plein d'emphase dénonce les dangers de la perte de l'identité culturelle et des traditions à cause de l'émigration massive et de l'arrivée de touristes qui s'installent sur l'île. Sa critique sociale et politique n'est certes pas dénuée de pertinence, mais elle est exagérément violente et réactionnaire. Sa rhétorique grandiloquente, adressée exclusivement aux hommes de la communauté, fonctionne finalement comme une caricature :

Chaque jour que Dieu fait, des hommes, des femmes quittent notre pays et s'en vont fouler le dur pavé des villes de la métropole. C'est là qu'ils se marient et font des enfants de « deuxième génération » comme on les appelle à présent qu'ils ne savent plus parler le créole ni danser le gwo ka. Pendant ce temps-là, toutes qualités de Blancs nous envahissent, engrossent nos femmes et nos filles, créent des enfants qui eux aussi ne savent plus à quelle terre ils appartiennent. Dans ces conditions, notre art de quimboiseur risque donc de disparaître. Les secrets transmis de père en fils risquent de se perdre. (28-29)

Dans ce discours nationaliste, « nos femmes et nos filles » sont des propriétés privées. De plus, l'exclusion des femmes de l'art du quimbois ("secrets transmis de père en fils ») est d'autant plus ridicule que la plupart des magiciennes puissantes sont des femmes dans les romans des autrices guadeloupéennes, que l'on songe à Man Cia dans Pluie et vent sur Télumée Miracle de Simone SchwarzBart ou Tituba dans Moi, Tituba, sorcière... noire de Salem de Maryse Condé. En outre, le nom même de l'école d'Agénor, École de la Connaissance Suprême, donne à comprendre qu'il est un charlatan prétentieux et vain.

Dans une atmosphère aussi patriarcale, Bella apparaît uniquement comme l'objet du désir des hommes. Comme son prénom le suggère, sa beauté est sa caractéristique principale : " elle était jolie à croquer, avec ses cheveux noirs et bouclés, sa peau de sapotille et ses yeux veloutés » (19). Les comparaisons qui assimilent la peau des femmes afro-caribéennes et surtout des femmes métisses à un fruit tropical comme la sapotille abondent dans les chansons populaires, la littérature et la poésie des Antilles ou sur les Antilles, que l'on songe par exemple au roman de Michèle Lacrosil Sapotille et le serin d'argile (1960) et son héroïne éponyme. De plus, bien que Bella soit une jeune femme des années 2000, l'illustration de couverture [voir fig.1] - très probablement un choix éditorial - la représente pieds nus, vêtue d'une robe traditionnelle et d'un foulard en madras, parée de colliers, de grandes boucles créoles et portant un panier 
de fruits tropicaux. Elle se tient devant une plage aux eaux turquoise avec palmiers et grand ciel bleu. Cette image comme figée dans le temps évoque immédiatement le cliché exotique et colonial de la femme créole ou de la « doudou » antillaise. Objet de tous les regards et de toutes les convoitises, Bella correspond d'une certaine manière au stéréotype de la belle " mulâtresse » associée à une forme d'érotisation et de fascination ou fétichisation sexuelle. Dans La Matrice de la race. Généalogie sexuelle et coloniale de la nation, Elsa Dorlin montre comment les traités de médecine de l'époque coloniale, empreints d'idéologie raciale et misogyne, associaient les femmes esclaves et surtout les « mulâtresses » à la séduction et à la débauche, pour mieux justifier et passer sous silence les violences sexuelles dont elles étaient victimes : "Le corps de la "mulâtresse" est par nature voué à la prostitution [...] [L]e destin fatal de la "mulâtresse" est celui de la courtisane. [...] La prétendue stérilité des "mulâtresses", comme celle des prostituées, est un effet de la lascivité, de 1'“ardeur" physique et morale qu'on leur prête. » (272-273).

De nos jours encore, dans la culture populaire, la « métisse » des tropiques est l'objet de tous les fantasmes exotiques, tout sourire sur les cartes postales pour touristes, associée à l'idée d'indolence et de sensualité. Or, de la même façon que Madame Zéphyr dans Rêves amers est une « mulâtresse à la peau couleur de miel » décrite comme une très belle femme, c'est justement en raison de son teint plus clair et de ses cheveux bouclés (c'est-à-dire non crépus) que Bella est considérée par tous comme la plus belle fille du village. Frantz Fanon a décrit dans Peau noire, masques blancs l'association entre beauté et blancheur comme un effet pervers du complexe d'infériorité et du racisme intériorisé que les populations colonisées développent au contact de la domination européenne. De ce point de vue, le succès de Bella est symptomatique de l'aliénation du sujet colonisé face à des standards de beauté directement liés à la hiérarchie par la couleur qui persiste dans la société caribéenne. Plus que sa beauté, c'est bien son ascendance blanche et européenne qui explique le mépris de Bella envers Carmélien et le reste de la communauté : « À cause de cette origine, Bella avait toujours considéré Sangre Grande comme un lieu indigne de sa beauté et de son intelligence. Elle ne rêvait que d'aller en Italie, pays de son défunt père. » (58)

À la fin du roman, la jeune femme rend visite à sa famille paternelle, mais expérimente à son tour le racisme. Elle qui se croyait blanche en Guadeloupe se découvre noire en Italie :

Elle n'avait pas eu de mal à retrouver Gorgonza, le village d'origine de son père, situé dans un cadre admirable sur les rives du lac de Côme. Mais, déception, la famille Servandoni l'avait fort mal reçue car visiblement elle n'appréciait pas les métis :

- Nous ne savions pas que Gian Carlo avait eu une fille, lui déclarait-on, avec mépris.

Les innombrables tantes, oncles, cousins, cousines défilaient devant elle en l'examinant avec curiosité et déclaraient pour conclure :

- Tu ne ressembles pas du tout à une Servandoni. Dans la famille nous sommes tous blonds avec les yeux verts. (84)

Même si, on l'a dit, les parents de Bella forment un couple marié, la réaction de la famille est la même que face à un enfant « bâtard », c'est-à-dire illégitime, thème récurrent de la littérature caribéenne. C'est le cas de Desirada : Marie-Noëlle pense qu'elle est la fille cachée que Gian Carlo Coppini, un homme marié à une Italienne, a eue avec la fille de sa domestique. Dans ce roman pour les adultes, au scandale qui entourerait la naissance d'un enfant issu d'un adultère s'ajoute le poids historique de la domination de race et de classe. Loin de considérer le métissage comme un enrichissement culturel, la famille italienne 
dans le roman pour la jeunesse perçoit la relation entre Gian Carlo et une femme afro-descendante de Guadeloupe comme indécente et déshonorante, comme s'il s'agissait d'une sulfureuse maîtresse. En refusant de traiter la mère de Bella comme leur belle-fille, les Servandoni replacent implicitement cette union dans la longue lignée des relations interdites, mais généralisées entre les maîtres blancs et leurs esclaves $^{10}$.

On observe donc que, même dans sa littérature de jeunesse, Maryse Condé refuse de céder à la célébration naïve du multiculturalisme. Le thème du métissage ne sert pas à faire l'éloge de la diversité comme on pourrait s'y attendre dans un roman pour les plus jeunes. La double origine de Bella symbolise au contraire les hiérarchies sociales et raciales et les blessures profondes de la société caribéenne postcoloniale.

\section{CONCLUSION}

Les enjeux spécifiques de la condition féminine et surtout les situations de violences sexuelles s'effacent dans les romans pour la jeunesse de Maryse Condé, où les protagonistes peuvent être indifféremment des jeunes filles ou des jeunes garçons. Cependant, les luttes et les difficultés des femmes forment souvent la toile de fond des récits où se dessinent les silhouettes de mères épuisées, de femmes bafouées, et même de femmes qui reproduisent l'oppression raciale et sociale sur d'autres femmes. Dans sa littérature de jeunesse comme dans ses plus grands best-sellers pour les adultes, Maryse Condé déjoue les attentes et fuit les oppositions binaires. Elle dépeint la misogynie ambiante et la vie des femmes comme elle est, « sans fards ».

\footnotetext{
${ }^{10}$ On trouve un écho de ce schéma familial avec le personnage de Mira dans un autre roman bien connu de Maryse Condé, Traversée de la mangrove. Orpheline d'une mère qu'elle n'a jamais connue, Mira est le fruit de l'union perçue comme honteuse entre un homme blanc, Loulou Lameaulnes, et une femme noire, Rosalie Sorane, morte en couches. Mira - celle que l'on admire - est connue dans tout le village pour sa grande beauté. Elle est l'objet du désir de tous les hommes, et sera même courtisée par un dénommé... Carmélien. Comme Bella, elle tire vanité de sa beauté et de sa peau claire et se considère comme un être d'exception : «Sans doute que je suis trop belle pour leur laideur, trop claire pour la noirceur de leurs peaux et de leurs cœurs. » (59).
} 


\section{BIBLIOGRAPHIE}

Alexander, Simone A. James. Mother Imagery in the Novels of Afro-Caribbean Women. University of Missouri, 2001.

Alibar, France, Pierrette Lembeye-Boy. Le couteau seul... Sé Kouto sèl... : La condition féminine aux Antilles. Vies de femmes, vol. 2. Éditions Caribéennes, 1990.

Brugier, Julie. «"Une féministe! Qu'est-ce que c'est que cela?” Penser le féminisme et l'écriture féminine dans Moi, Tituba sorcière... Noire de Salem (1986) et Célanire cou-coupé (2001) de Maryse Condé ». SELF XX-XXI, Actes de la journée d'études "Écriture féminine aux XXe et XXIe siècles, entre stéréotype et concept » (12/12/2015). https://self.hypotheses.org/publicationsen-ligne/ecriture-feminine-aux-xxe-et-xxie-siecles-entre-stereotype-et-concept/rapportsambivalents-a-lheritage-feministe-2

Condé, Maryse. La Belle et la Bête, une version guadeloupéenne. Larousse, 2013.

—. «Entretien avec Maryse Condé ». Hommes \& migrations, no. 1301, 2013, par Marie Poinsot et Nicolas Treiber. http://journals. openedition.org/hommesmigrations/1953.

—. Savannah Blues. Sépia, 2009.

-. Chiens fous dans la brousse. Bayard Jeunesse, 2008.

—. Rêves amers. Bayard Jeunesse, 2001.

—. Desirada. Robert Laffont, 1997.

-. Hugo le terrible. Sépia, 1991.

-. «Mode d'emploi : Comment devenir une écrivaine que l'on dit antillaise ». Nouvelles Études Francophones, vol. 22 no. 1, 2007. http://www.jstor.org/stable/25702014.

—. Traversée de la mangrove. Mercure de France, 1989.

—. Moi, Tituba, sorcière... Noire de Salem. Mercure de France, 1986.

—. Ségou : Les murailles de terre. Robert Laffont, 1984.

—. La Parole des femmes : Essai sur les romancières des Antilles de langue française. L'Harmattan, 1979.

Chartres, Fanny. Solaire. L'École des Loisirs, 2018.

Crenshaw, Kimberle Williams. « Mapping the Margins: Intersectionality, Identity Politics, and Violence Against Women of Color ». The Feminist Philosophy Reader, édité par Alison Bailey and Chris Cuomo, McGraw-Hill, 2008, pp. 279-309.

Dorlin, Elsa. La Matrice de la race. Généalogie sexuelle et coloniale de la nation. La Découverte, 2006.

Fanon, Frantz. Peau noire, masques blancs. Seuil, 1952.

Franchini, Pauline. « De Ségou à Chiens fous dans la brousse : un exemple d'auto-réécriture en version enfantine chez Maryse Condé ». Écrire pour la jeunesse et pour les adultes. D'un lectorat à 
l'autre, édité par Marion Mas and Anne-Marie Mercier-Faivre, Classiques Garnier, 2020, pp. 163-178.

Gyssels, Kathleen. Passes et impasses dans le comparatisme postcolonial caribéen. Cinq traverses. Champion, 2010.

Lacrosil, Michèle. Sapotille et le serin d'argile. Gallimard, 1960.

Neel, Julien. Lou! Journal infime. Glénat, 2004.

Parisot, Yolaine. «"Trouble dans le genre" caribéen, l'Autre du cosmopolitisme postcolonial ». Genre et migrations postcoloniales : lectures croisées de la norme, édité par Nadia Ouabdelmoumen et Yolaine Parisot, Presses Universitaires de Rennes, 2013, pp. 123-141.

Petersen, Kirsten Holst, Anna Rutherford. A Double Colonization: Colonial and Post-Colonial Women's Writing. Dangaroo, 1985.

Pineau, Gisèle. La grande drive des esprits. Stock, 1993.

Roch, Alexandra. « La déconstruction de la mère sacrée ou de la femme poto-mitan dans L'Amour à la créole et Je suis une Martiniquaise libertine de Pyrrha Ducalion » Les Cahiers du GRELCEF, no. 11, « La sexualité et ses tabous dans les fictions francophones », mai 2019, pp. 105-119.

Rody, Caroline. The Daughter's Return: African-American and Caribbean Women's Fictions of History. Oxford UP, 2001.

Schwarz-Bart, Simone. Pluie et vent sur Télumée Miracle. Seuil, 1972.

Thomas, Bonnie. Breadfruit or Chestnut? Gender Construction in the French Caribbean Novel. Lexington Books, 2006.

Zobel, Joseph. La Rue Cases-Nègres. Présence Africaine, 1950. 